\title{
Finite Element Approximation for Time-Dependent Diffusion with Measure-Valued Source
}

\author{
Thomas I. Seidman - Matthias K. \\ Gobbert · David W. Trott • Martin \\ Kružík
}

Received: date / Accepted: date

\begin{abstract}
The convergence of finite element methods for elliptic and parabolic partial differential equations is well-established if source terms are sufficiently smooth. Noting that finite element computation is easily implemented even when the source terms are measure-valued - for instance, modeling point sources by Dirac delta distributions - we prove new convergence order results in two and three dimensions both for elliptic and for parabolic equations with measures as source terms. These analytical results are confirmed by numerical tests using COMSOL Multiphysics.
\end{abstract}

Keywords Dirac delta distribution · Error estimates · Finite element approximation $\cdot$ Linear diffusion equation $\cdot$ Measure-valued data

Mathematics Subject Classification (2000) 35K57 -65M15 -65M60

\section{Introduction}

This paper is concerned with two related problems: the parabolic (time-dependent) diffusion problem

$$
u_{t}-\nabla \cdot D \nabla u=\varphi \quad \text { with }\left.u\right|_{t=0}=0
$$

Thomas I. Seidman, Matthias K. Gobbert, David W. Trott

Department of Mathematics and Statistics,

University of Maryland, Baltimore County, Baltimore, MD 21250, USA

E-mail: $\{$ seidman,gobbert,dtrott1\}@umbc.edu

Martin Kružík

Institute of Information Theory and Automation,

Academy of Sciences of the Czech Republic, CZ-182 08 Praha 8, Czech Republic and

Faculty of Civil Engineering,

Czech Technical University, CZ-166 29 Praha 6, Czech Republic,

E-mail: kruzik@utia.cas.cz 
on $\mathcal{Q}=\mathcal{Q}_{T}=[0, T] \times \Omega$ (with homogeneous boundary conditions and 0 initial state), which is our primary concern, and the corresponding steady state problem

$$
-\nabla \cdot D \nabla u=\varphi
$$

on $\Omega$ (again with homogeneous boundary conditions), restricting attention to autonomous linear problems so $D=D(x)$, etc.

Our focus will be on convergence order error estimates of the form

$$
\|u-U\|_{\mathcal{Y}} \leq K h^{\alpha}\|\varphi\|_{\mathcal{X}}
$$

where $u$ is the solution of (1) or (2), $U=U^{\mathcal{V}}$ is the corresponding finite element solution of each problem, using the finite element space $\mathcal{V}$, and $h=$ $h(\mathcal{T}) \rightarrow 0$ is the mesh parameter of the triangulation $\mathcal{T}$ determining $\mathcal{V}$. The convergence order estimate will always be expressed in terms of the regularity of the data $\varphi \in \mathcal{X}$, rather than that of the solution $u$. Thus (3) would be estimating approximation error in the space $\mathcal{Y}$ for data in the space $\mathcal{X}$ assuming, of course, that $\mathcal{V} \subset \mathcal{Y}$, that $\mathcal{X}$ embeds in the dual $\mathcal{V}^{*}$ and that the solution $u$ would be in $\mathcal{Y}$ for data in $\mathcal{X}$.

Throughout we will take $\mathcal{Y}$ to be $L^{2}(\Omega)$ for measuring approximation error for (2) and a space of spatially $L^{2}(\Omega)$-valued functions of $t$ for (1). One wellknown example of the form (3) (cf., e.g., [12]) is for $\varphi \in \mathcal{X}=L^{2}$ data:

$$
\text { One has } \alpha=2 \text { in (3) for (2) when } \mathcal{X}=L^{2}(\Omega), \mathcal{Y}=L^{2}(\Omega) \text {. }
$$

The use of finite element spaces of continuous piecewise affine functions certainly admits the formal possibility of using the Finite Element Method (FEM) for such problems with measure data and our concern here is to justify and analyze this formal procedure.

Our own contact with these problems started when modeling calcium flow in heart cells, where the injection of calcium ions at locations throughout the interior of the cell is modeled as point sources represented by Dirac delta distributions; see [8], [5] and references therein for the application background. In both of these papers, heuristic arguments and numerical evidence for timedependent problems of the form (1) suggest estimates of the form (3) with $\alpha \approx 2-d / 2$ for domains $\Omega \subset \mathbb{R}^{d}$; one version of that numerical evidence will be shown in Figure 1.

Thus, the purpose of this paper is to prove rigorous results of the form (3) for cases when the source term $\varphi$ in (2) is not a function in $L^{2}$, but is permitted to be a delta function or, somewhat more generally, a measure $\mu$ in $\mathcal{M}=[C(\bar{\Omega})]^{*}$. There has been considerable interest recently in such equations and their numerical solution. See, e.g., [10], [4], [1], etc., but one may go farther back, almost 40 years, to Ridgway Scott [11]. The papers [4], [1] are not quite comparable with our present concerns and, despite its title, [2] does not consider measure-valued data. Most significantly, we note that [11] gives an $\mathcal{O}\left(h^{2-d / 2}\right)$ error estimate for a single delta function as the right-hand side, but the relevant result there is not in the form (3) and does not apply directly to more general source terms. 
We will also be concerned to get estimates for finite element approximation of (1). Indeed, we are thinking of the time-dependent problem (1) as our primary interest. The source term $\varphi$ in (1) would then be a time-dependent $\mathcal{M}$-valued function $\mu(t)$ with results depending on our assumptions for its $t$ variation. The equations (1) and (2) are closely related structurally and it seems appropriate to consider them together and, in fact, to treat the more easily handled (2) first. Our arguments will largely follow [11] in spirit with the use of [12] and semigroup theory for the parabolic problem.

This paper is organized as follows: Section 2 states the formulation and defines the notation used throughout. Sections 3 and 4 analyze the stationary problem (2), with the main new result in Theorem 4.2. Section 5 provides the main result for the time-dependent problem (1) in Theorem 5.1. Finally, Section 6 presents the computational studies that accompany the analytical results.

\section{Formulation and notation}

For (2) with homogeneous Dirichlet boundary conditions one considers the solution $u$ as satisfying the weak form

$$
\langle\nabla v, D \nabla u\rangle=\langle v, \varphi\rangle \quad \text { for all admissible } v
$$

and, given a finite element space $\mathcal{V}$ (e.g., of piecewise affine functions on some triangulation $\mathcal{T}$, vanishing at the boundary), the finite element Galerkin approximation $U \in \mathcal{V}$ is obtained as satisfying

$$
\langle\nabla v, D \nabla U\rangle=\langle v, \varphi\rangle \quad \text { for all } v \in \mathcal{V} \text {. }
$$

For simplicity we will assume throughout that the spatial region $\Omega \subset \mathbb{R}^{d}$ is open, bounded and connected with adequately regular boundary $\partial \Omega$. We further assume that $d \leq 3$ and that the diffusion coefficient $D$ is smooth enough to ensure that for (2) with homogeneous Dirichlet conditions

We have $u \in H^{2}(\Omega) \cap H_{0}^{1}(\Omega)$ for data $\varphi \in L^{2}(\Omega)$.

$$
H^{2}(\Omega) \cap H_{0}^{1}(\Omega) \subset C^{0, \lambda}(\bar{\Omega}) \text { for (some) } 0<\lambda<2-d / 2 \text {. }
$$

[Here $H^{2}, H_{0}^{1}$ are the usual Sobolev spaces and $C^{0, \lambda}$ is the Hölder space with exponent $0<\lambda<1$.] By standard elliptic regularity results and the Sobolev Embedding Theorem, we note that this is effectively a mild geometric assumption on the regularity of $\partial \Omega$ supplemented, e.g., by requiring $D \in W^{1, \infty}(\Omega)$ as well as uniform ellipticity. What we will actually use is a consequence of this: that the map: $\varphi \mapsto u$ is continuous from $L^{2}(\Omega)$ to $C^{0, \lambda}(\bar{\Omega})$ so, by duality and noting the divergence form of the equation (2), we have

$$
\mathbf{S}: \mathcal{M}^{\lambda}(\Omega)=\left[C^{0, \lambda}(\bar{\Omega})\right]^{*} \rightarrow L^{2}(\Omega) \text { is continuous, i.e., }\|\mathbf{S}\|<\infty
$$

for the solution operator $\mathbf{S}$ of (2). 
Throughout this paper, for our concern with spatial discretization we will let $\mathcal{T}$ denote a regular partition of the region $\Omega$ and will let $\mathcal{V}$ denote the associated space of continuous piecewise affine functions. Thus we assume that the region $\Omega$ is to be partitioned by a triangulation $\mathcal{T}$, consisting of simplices $E$ with compatible faces; the mesh parameter $h=h(\mathcal{T})=\max _{E \in \mathcal{T}}\{\operatorname{diam}(E)\}$ then indicates the scale of the triangulation. The notion of regularity here means that the elements have roughly the same shape as the reference simplex $E_{\ddagger}$ and, for each value $h>0$ of the scale parameter are of roughly the same size, taking $\operatorname{diam}\left(E_{\ddagger}\right)=1$. We make this precise by assuming that: for each $E \in \mathcal{T}$ there is an affine map of the form:

$$
\psi=\psi_{E}: E_{\ddagger} \rightarrow E: x_{\ddagger} \mapsto x=\mathrm{v}+h A_{E} x_{\ddagger}
$$

with $h=h(\mathcal{T})$ and all the matrices $\left\{A_{E}\right\}$ restricted to some fixed compact set $\mathfrak{A} \subset G L(d)$. We then denote by $\mathfrak{T}=\mathfrak{T}_{\mathfrak{A}}$ the family of all such triangulations with the same set $\mathfrak{A}$ and let $\mathfrak{T}_{h}=\{\mathcal{T} \in \mathfrak{T}: h(\mathcal{T}) \leq h\}$.

Corresponding to each such triangulation $\mathcal{T} \in \mathfrak{T}$ is the space $\mathcal{W}=\mathcal{W}(\mathcal{T})$ consisting of all functions on $\Omega$ which are affine on each simplex $E \in \mathcal{T}$. The finite element space under consideration is then $\mathcal{V}=\mathcal{W} \cap C_{0}(\Omega)$, noting the homogeneous Dirichlet boundary conditions. It will also be convenient to let $\mathcal{W}(E)$ be the $(d+1)$-dimensional space of affine functions on $E$ for each $E \in \mathcal{T}$, so $\mathcal{W}(E)=\psi_{E}^{-1} \mathcal{W}\left(E_{\ddagger}\right)$ and $\mathcal{W}(\mathcal{T})=\oplus \mathcal{W}(E)$.

No norm is specified for $\mathcal{V}$ or $\mathcal{W}(\mathcal{T})$, but when desired we may use appropriate subscripts to indicate use of a specified norm: e.g., we write $\mathcal{W}=\mathcal{W}_{p}(\mathcal{T})$ for use of the $L^{p}(\Omega)$-norm $\|\cdot\|_{p}$. We will write $\|\cdot\|$ for the $L^{2}$-norm, identifying other norms by appropriate subscripts.

In our estimations we will use $K$ to denote an arbitrary positive constant (replaceable by any larger constant and not necessarily the same at each appearance) which may depend on $d, \Omega, \mathfrak{A}, \ldots$ or prior instantiations of $K$, but does not depend on any particular $\varphi, u, \mathcal{V}$, etc., and specifically does not depend on the mesh parameter $h$ except that it may require that $h>0$ be 'sufficiently small'. It follows from the regularity assumption that we have

$$
\left\|A_{E}\right\| \leq K, \quad 1 / K \leq\left|\operatorname{det} A_{E}\right| \leq K, \quad h / K \leq \operatorname{diam}(E) \leq K h
$$

for all $E \in \mathcal{T}$ as consequences of the compactness of $\mathfrak{A}$.

\section{An elliptic estimate based on [11]}

We begin here with the relevant estimate from [11].

Theorem 3.1 [Scott] Let $\Omega \subset \mathbb{R}^{d}$ with $d=2,3$ and let $\varphi=\delta_{\xi}$, a delta function located at the point $\xi \in \Omega$. Let $\mathcal{V}$ be the finite element space of piecewise affine elements on a regular triangulation $\mathcal{T}$. Then, for each $\xi \in \Omega$ there is a 
function $f=f^{\xi} \in L^{2}(\Omega)$ satisfying

$$
\begin{aligned}
& \text { a) } \quad\langle f, \Phi\rangle=\left\langle\delta_{\xi}, \Phi\right\rangle=\Phi(\xi) \text { for all } \Phi \in \mathcal{V} \subset \mathcal{W}(\mathcal{T}) \\
& \text { b) } \quad\|f\|_{L^{2}(\Omega)} \leq c(\xi) h^{-d / 2} \\
& \text { c) } \quad\left\|f-\delta_{\xi}\right\|_{\mathcal{X}^{-}} \leq K h^{\alpha_{*}} \text { with } \alpha_{*}=2-d / 2 \text {. }
\end{aligned}
$$

Letting $u=u^{\xi}$ be the solution of (2) with $\varphi=\delta_{\xi}$ and $U$ be the FEM approximation, satisfying (6), one then has

$$
\|u-U\| \leq c(\xi) h^{\alpha_{*}} .
$$

The auxiliary space $\mathcal{X}^{-}$used in the paper [11] is the space $\Xi^{-2}$ defined in [9]. It is the dual space to

$$
\Xi^{2}=\left\{v \in L^{2}(\Omega):\|v\|_{\Xi^{2}}=\sum_{|\alpha| \leq 2}\left\|\varrho^{|\alpha|} \frac{\partial^{|\alpha|} v}{\partial x_{1}^{\alpha_{1}} \cdots \partial x_{d}^{\alpha_{d}}}\right\|<\infty\right\},
$$

where $\varrho$ is a smooth function on $\Omega$ satisfying

$$
c_{1} \operatorname{dist}(\xi, \partial \Omega) \leq \varrho(\xi) \leq c_{2} \operatorname{dist}(\xi, \partial \Omega)
$$

for some $c_{2}>c_{1}>0$. We note that the asymptotics

$$
c(\xi)=\mathcal{O}\left(\operatorname{dist}(\xi, \partial \Omega)^{-2}\right) \quad \text { as } \operatorname{dist}(\xi, \partial \Omega) \rightarrow 0
$$

of $c(\xi)$ in (11) comes from this choice; cf. [11]. Of course, to have $\alpha_{*}>0$ we need the dimension $d$ to be less than 4 .

Since $c(\xi) \rightarrow \infty$ as $\operatorname{dist}(\xi, \partial \Omega) \rightarrow 0$, we cannot expect to deduce from this an $\mathcal{O}\left(h^{\alpha_{*}}\right)$ estimate of the form $(3)$ with $\mathcal{X}=\mathcal{M}$. Instead, we may introduce the $c$-weighted norm

$$
\|\mu\|_{c}=\int_{\Omega} c(x)|\mu|(d x) .
$$

and consider as the space of source terms only those signed measures for which this norm is finite.

Theorem 3.2 Let $\mathcal{X}$ be the the space $\mathcal{M}_{c}=\left\{\mu \in \mathcal{M}(\Omega):\|\mu\|_{c}<\infty\right\}$. Then one has the $\mathcal{O}\left(h^{2-d / 2}\right)$ convergence order, i.e., with $\alpha_{*}=2-d / 2$ one has

$$
\|u-U\| \leq h^{\alpha_{*}}\|\varphi\|_{\mathcal{X}} \quad \text { for source terms } \varphi=\mu \in \mathcal{X}=\mathcal{M}_{c}(\Omega) .
$$

ProOF: $\quad$ Write $\tilde{u}=\tilde{u}(\xi, \cdot)$ for the solution $u$ with $\varphi=\delta_{\xi}$ as $\xi$ varies over $\Omega$ and observe that $(\xi, x) \mapsto \tilde{u}(\xi, x)$ is then just the fundamental solution of the system. In much the same way we have $\tilde{U}(\xi, \cdot)$ as the corresponding FEM solution, satisfying (6) with finite element space $\mathcal{V}$.

As is standard, linearity gives $\int \tilde{u}(\xi, x) \varphi(\xi) d \xi$ as the solution $\mathbf{S} \varphi$ of (2) for, e.g., smooth $\varphi$. We have continuity of $\mathbf{S}: \mathcal{M} \rightarrow \mathcal{Y}=L^{2}(\Omega)$ so, by the density in $\mathcal{M}$ of such smooth functions, we have the representations

$$
u=\mathbf{S} \mu=\int_{\Omega} \tilde{u}(\xi, \cdot) \mu(d \xi), \quad U=\int_{\Omega} \tilde{U}^{\mathcal{V}}(\xi, \cdot) \mu(d \xi)
$$


for $u$ and $U$ satisfying (2) and (6), respectively. Using (11), this gives

$$
\begin{aligned}
\|u-U\| & =\left\|\int_{\Omega}[\tilde{u}(\xi, \cdot)-\tilde{U}(\xi, \cdot)] \mu(d \xi)\right\| \\
& \leq \int_{\Omega}\left[c(\xi) h^{\alpha_{*}}\right]|\mu|(d \xi)=h^{\alpha_{*}}\|\mu\|_{\mathcal{M}_{c}} .
\end{aligned}
$$

which is just (14).

As a possibly useful corollary we obtain the following slightly simpler result (provided we can restrict attention to measures with support in a subset $\Omega^{\prime}$ compactly contained in $\Omega$ ).

Corollary 3.1 Fix $\Omega^{\prime} \Subset \Omega$ and let $\mathcal{M}\left(\Omega^{\prime}\right)=\left[C\left(\Omega^{\prime}\right)\right]^{*}$ be identified with the subspace $\left\{\mu \in \mathcal{M}: \mu(S)=0\right.$ for $\left.S \subset\left[\Omega \backslash \Omega^{\prime}\right]\right\}$ of measures in $\mathcal{M}=\mathcal{M}(\Omega)$ with support in $\Omega^{\prime}$, taking $\|\mu\|_{\mathcal{M}\left(\Omega^{\prime}\right)}=\|\mu\|_{\mathcal{M}}=\int_{\Omega^{\prime}}|\mu|(d \xi)$. Then one has

$$
\|u-U\| \leq K h^{2-2 / d}\|\varphi\|_{\mathcal{M}} \quad \text { for source terms } \varphi=\mu \in \mathcal{M}\left(\Omega^{\prime}\right) .
$$

Proof: Take $K=K_{\Omega^{\prime}}=\max \left\{c(\xi): \xi \in \Omega^{\prime}\right\}$ in replacing $\|\mu\|_{c}$ by $\|\mu\|_{\mathcal{M}\left(\Omega^{\prime}\right)}$; this gives $K<\infty$ as $\Omega^{\prime}$ since bounded away from $\partial \Omega$.

Remark 3.1 The use of the space $\Xi^{-2}$ from [9] also leaves a regularity gap since [9] assumes, for simplicity, that $\Omega$ has a $C^{\infty}$ boundary — yet any possibility of exact triangulation by simplices means that $\partial \Omega$ must be piecewise affine. In [11] this issue is parenthetically addressed by the suggestion that one might consider the use of elements at the boundary with curved faces. In relying on Theorem 3.1 to obtain Theorem 3.2 we are conforming to the treatment in [11].

\section{An alternative approximation theorem}

Our goal in this section is to prove another approximation result, now applying to more general measures $\mu \in \mathcal{M}$ with approximation by elements of the space $\mathcal{V} \subset \mathcal{W}(\mathcal{T})$ of continuous piecewise affine functions on a regular triangulation $\mathcal{T}$ of $\Omega$. The properties (17) of the selection $\Pi^{\mathcal{V}}: \mu \mapsto f$ will be comparable to (10) for the approximation of $\delta_{\xi}$ by an $L^{2}$ function used in [11] and we also apply this to get a convergence order for the elliptic (steady state) problem of the form (3) for $\mathcal{X}=\mathcal{M}$. Now relying on the assumption (7), the key distinction between this construction and that of Theorem 3.1 is the replacement of the auxiliary space $\mathcal{X}^{-}=\Xi^{-2}$ from [9] used there by the use here of the dual of a Hölder space, taking $\mathcal{X}^{-}=\mathcal{M}^{\lambda}(\Omega)=\left[C^{0, \lambda}(\Omega)\right]^{*}$, for which see (21). The resulting Theorem 4.1 can then be used for estimating the FEM convergence order when $\mathcal{X}=\mathcal{M}$. 
Theorem 4.1 Take $0<\lambda<1$ as in (7) and $\mathcal{V}$ as above with $h=h(\mathcal{T})$. Then, for any measure $\mu \in \mathcal{M}$ there is a function $f=\Pi^{\mathcal{V}} \mu \in L^{2}(\Omega)$ satisfying

$$
\begin{gathered}
\text { a) }\langle f, \Phi\rangle=\langle\mu, \Phi\rangle \quad \text { for all } \Phi \in \mathcal{V} \subset \mathcal{W}(\mathcal{T}) \\
\text { b) } \quad\|f\|_{L^{2}(\Omega)} \leq K h^{-d / 2}\|\mu\|_{\mathcal{M}} \\
\text { c) } \quad\|f-\mu\|_{\mathcal{X}^{-}} \leq K h^{\lambda}\|\mu\|_{\mathcal{M}}
\end{gathered}
$$

now with $\mathcal{X}^{-}=\mathcal{M}^{\lambda}(\Omega)=\left[C^{0, \lambda}(\Omega)\right]^{*}$ where $C^{0, \lambda}(\Omega)$ is the space of Hölder continuous functions with exponent $\lambda$.

We begin with the observation that, for any triangulation $\mathcal{T}$ and for any $1 \leq p<\infty$ one has $L^{p}(\Omega)=\oplus L^{p}(E)$ : for any function $f \in L^{p}(\Omega)$ there are (unique) functions $f_{E}=\left.f\right|_{E} \in L^{p}(E)$ for $E \in \mathcal{T}$ and, using (9), one has

$$
\begin{aligned}
\|f\|_{L^{p}(\Omega)}^{p} & =\int_{\Omega}|f(x)|^{p} d x=\sum_{E \in \mathcal{T}} \int_{E}\left|f_{E}\right|^{p} d x \\
& =\sum_{E \in \mathcal{T}} \int_{E_{\ddagger}}\left|f_{E} \circ \psi\right|^{p}\left|\operatorname{det}\left(\psi^{\prime}\right)\right| d x_{\ddagger} \\
& =h^{d} \sum_{E \in \mathcal{T}}\left|\operatorname{det}\left(A_{E}\right)\right|\left\|f_{E} \circ \psi\right\|_{L^{p}\left(E_{\ddagger}\right)}^{p} \\
\text { so: } \quad\|f\|_{L^{p}(\Omega)} & \leq K h^{d / p} \sum_{E \in \mathcal{T}}\left\|f_{E} \circ \psi\right\|_{L^{p}\left(E_{\ddagger}\right)}
\end{aligned}
$$

Having introduced the space of (signed) measures $\mathcal{M}=[C(\Omega)]^{*}$, we now also note that one can analogously decompose each measure $\mu \in \mathcal{M}$ as

$$
\mu=\sum_{E} \mu_{E} \quad\|\mu\|_{\mathcal{M}(\Omega)}=\sum_{E \in \mathcal{T}}\left\|\mu_{E}\right\|_{\mathcal{M}(\Omega)} \quad \operatorname{supp}\left(\mu_{E}\right) \subset E .
$$

[This decomposition is almost unique: we note, however, that a bit of selection may be needed in case $|\mu|$ might assign nontrivial measure to some subset of a face $E \cap E^{\prime}$, requiring that this be split, somewhat arbitrarily, between $\mu_{E}$ and $\mu_{E^{\prime}}$. This consideration is relevant only to the analysis, not to the computation.] Note that each $\mu_{E}$ is in $\mathcal{M}=\mathcal{M}(\Omega)$, but can also be viewed in $\mathcal{M}(E)=[C(E)]^{*}$ with $\left\|\mu_{E}\right\|_{\mathcal{M}(E)}=\left\|\mu_{E}\right\|_{\mathcal{M}}$ since each $\varphi \in C(E)$ can be extended to $\Omega$ without increase in norm. We also note that we may use (9) here again to get

$$
\begin{aligned}
\left\|\mu_{E}\right\|_{\mathcal{M}(E)} & =\int_{E}\left|\mu_{E}\right| d x=\int_{E_{\ddagger}}\left|\operatorname{det}\left(\psi^{\prime}\right)\right|\left|\mu_{E} \circ \psi\right| d x_{\ddagger} \\
& =h^{d}\left|\operatorname{det}\left(A_{E}\right)\right|\left\|\mu_{E} \circ \psi\right\|_{\mathcal{M}\left(E_{\ddagger}\right)}
\end{aligned}
$$

For the Hölder space $C^{0, \lambda}(\Omega)$ for $0 \leq \lambda<1$ and its dual $\mathcal{M}^{\lambda}=\mathcal{M}^{\lambda}(\Omega)=$ $\left[C^{0, \lambda}(\Omega)\right]^{*}$ we use the norms

$$
\|\varphi\|_{C^{0, \lambda}(\Omega)}=\sup _{x \in \Omega}\{|\varphi(x)|\}+\sup _{x \neq y \in \Omega}\left\{\frac{|\varphi(x)-\varphi(y)|}{|x-y|^{\lambda}}\right\}
$$


with the corresponding dual norm for $\mu \in \mathcal{M}^{\lambda}$

$$
\|\mu\|_{\mathcal{M}^{\lambda}}=\|\mu\|_{\left[C^{0, \lambda}(\Omega)\right]^{*}}=\sup \left\{\langle\mu, \varphi\rangle:\|\varphi\|_{C^{0, \lambda}(\Omega)} \leq 1\right\} .
$$

Clearly $C^{0, \lambda}(\Omega) \hookrightarrow C(\Omega)$ so, by duality, $\mathcal{M} \hookrightarrow \mathcal{M}^{\lambda}$. [If $\mu \in \mathcal{M}$ is decomposed as in (19), then each $\mu_{E}$ is in $\mathcal{M}^{\lambda}(\Omega)$, but can also be viewed in $\mathcal{M}^{\lambda}(E)=\left[C^{0, \lambda}(E)\right]^{*}$. Note that $\left\|\mu_{E}\right\|_{\mathcal{M}^{\lambda}(E)}=\left\|\mu_{E}\right\|_{\mathcal{M}^{\lambda}(\Omega)}$ since, again, each $\varphi \in C^{0, \lambda}(E)$ can be extended to $\Omega$ without increase in $C^{0, \lambda}$-norm.] With these preliminaries we are ready to prove Theorem 4.1.

Proof:

Step 1: We begin by decomposing $\mu$ as in (19) and considering each $E$ separately. Each $\mathcal{W}(E)$ is a finite dimensional subspace of $C(E)$ and we let $\tilde{\mu}_{E}$ be the restriction of $\mu_{E}$ to $\mathcal{W}(E)$ so an element of $[\mathcal{W}(E)]^{*}$. In considering $\mathcal{W}(E)$ as a subspace of $C(E)$ we are implicitly using the sup-norm for $\mathcal{W}(E)$ - denoting this by $\mathcal{W}_{\infty}(E)$ - and observe that, as a restriction, we have

$$
\left\|\tilde{\mu}_{E}\right\|_{\left[\mathcal{W}_{\infty}(E)\right]^{*}} \leq\left\|\mu_{E}\right\|_{[C(E)]^{*}}=\left\|\mu_{E}\right\|_{\mathcal{M}}
$$

Since $\mathcal{W}(E)$ is finite dimensional, there is then a unique $f_{E} \in \mathcal{W}(E)-$ i.e., with $\operatorname{supp}\left(f_{E}\right) \subset E-$ such that

$$
\left\langle\mu_{E}, w\right\rangle=\int_{E} f_{E} w d x \quad \text { for all } w \in \mathcal{W}(E) .
$$

[Given any basis $\left\{b_{j}\right\}$ for $\mathcal{W}(E)$, we may find coefficients $\alpha_{j k}$ such that

$$
\int_{E}\left(\sum_{k} \alpha_{j k} b_{k}\right) b_{j} d x=\left\langle\tilde{\mu}_{E}, b_{j}\right\rangle
$$

for each $j$.] If we were to use the $L^{2}(E)$-norm for $\mathcal{W}(E)$, getting $\mathcal{W}_{2}(E)$, we could equally well consider $\tilde{\mu}_{E}$ as an element of $\left[\mathcal{W}_{2}(E)\right]^{*}$ since $\mathcal{W}_{2}(E)$ is a Hilbert space: we then get

$$
\left\|f_{E}\right\|_{\mathcal{W}_{2}(E)}=\left[\int_{E}\left|f_{E}\right|^{2} d x\right]^{1 / 2}=\left\|\tilde{\mu}_{E}\right\|_{\left[\mathcal{W}_{2}(E)\right]^{*}}
$$

Now define $f \in L^{2}(\Omega)$ as $f=\sum_{E} f_{E}$ giving $f \in \mathcal{W}(\mathcal{T})=\oplus \mathcal{W}(E)$. For any $\Phi \in \mathcal{W}(\mathcal{T})$, decomposed as $\Phi=\sum \Phi_{E}$, one then has

$$
\langle\mu, \Phi\rangle=\sum_{E \in \mathcal{T}}\left\langle\mu_{E}, \Phi_{E}\right\rangle=\sum_{E \in \mathcal{T}}\left\langle\tilde{\mu}_{E}, \Phi_{E}\right\rangle=\sum_{E \in \mathcal{T}} \int_{E} f_{E} \Phi_{E} d x=\int_{\Omega} f \Phi d x
$$

so we have $(17-a)$. 
Step 2: We have $\left\|\tilde{\mu}_{E}\right\|_{\left[\mathcal{W}_{\infty}(E)\right]^{*}} \leq\left\|\mu_{E}\right\|_{\mathcal{M}}$ and, using (24) and (9), we obtain

$$
\begin{aligned}
& \left\|\tilde{\mu}_{E}\right\|_{\left[\mathcal{W}_{\infty}(E)\right]^{*}} \\
& \quad=\sup \left\{\int_{E} f_{E} w d x: w \in \mathcal{W}(E),|w| \leq 1\right\} \\
& =h^{d}\left|\operatorname{det}\left(A_{E}\right)\right| \sup \left\{\int_{E}\left[f_{E} \circ \psi\right][w \circ \psi] d x: w \circ \psi \in \mathcal{W}\left(E_{\ddagger}\right),|w| \leq 1\right\} \\
& =h^{d}\left|\operatorname{det}\left(A_{E}\right)\right|\left\|f_{E} \circ \psi\right\|_{\left[\mathcal{W}_{\infty}\left(E_{\ddagger}\right)\right]^{*}}
\end{aligned}
$$

while, for comparison, we have

$$
\left\|f_{E}\right\|_{\mathcal{W}_{2}(E)}=\left[\int_{E}\left|f_{E}\right|^{2} d x\right]^{1 / 2}=h^{d / 2}\left|\operatorname{det}\left(A_{E}\right)\right|^{1 / 2}\left\|f_{E} \circ \psi\right\|_{\mathcal{W}_{2}\left(E_{\ddagger}\right)} .
$$

Since $\mathcal{W}\left(E_{\ddagger}\right)$ is a fixed finite dimensional space, we note that all norms on it are equivalent so there is a constant $K$ such that

$$
\|w\|_{\mathcal{W}_{2}\left(E_{\ddagger}\right)} \leq K\|w\|_{\left[\mathcal{W}_{\infty}\left(E_{\ddagger}\right)\right]^{*}} \quad \text { for all } w \in \mathcal{W}\left(E_{\ddagger}\right) .
$$

Note that $\left[f_{E} \circ \psi\right]=w \in \mathcal{W}\left(E_{\ddagger}\right)$ in each of our cases so

$$
\begin{aligned}
\left\|f_{E}\right\|_{\mathcal{W}_{2}(E)} & =h^{d / 2}\left|\operatorname{det}\left(A_{E}\right)\right|^{1 / 2}\left\|f_{E} \circ \psi\right\|_{\mathcal{W}_{2}\left(E_{\ddagger}\right)} \\
& \leq h^{d / 2}\left|\operatorname{det}\left(A_{E}\right)\right|^{1 / 2} K\left\|f_{E} \circ \psi\right\|_{\left[\mathcal{W}_{\infty}\left(E_{\ddagger}\right)\right]^{*}} \\
& =h^{-d / 2}\left|\operatorname{det}\left(A_{E}\right)\right|^{-1 / 2} K\left\|\tilde{\mu}_{E}\right\|_{\left[\mathcal{W}_{\infty}(E)\right]^{*}} \leq K h^{-d / 2}\left\|\mu_{E}\right\|_{\mathcal{M}} .
\end{aligned}
$$

The triangle inequality then gives $(17-b)$.

Step 3: For (17-c) we again proceed for each $E$ separately. We begin, somewhat similarly to the above, by noting that

$$
\begin{aligned}
& \left\|f_{E}\right\|_{\mathcal{M}}=\sup \left\{\int_{E} f_{E} w d x: w \in C(E),|w| \leq 1\right\} \\
& \leq\left\|f_{E}\right\|_{\mathcal{W}_{1}(E)} \leq h^{d}\left|\operatorname{det}\left(A_{E}\right)\right|\left\|f_{E} \circ \psi\right\|_{\mathcal{W}_{1}\left(E_{\ddagger}\right)} \\
& \leq h^{d}\left|\operatorname{det}\left(A_{E}\right)\right| K\left\|f_{E} \circ \psi\right\|_{\left[\mathcal{W}_{\infty}\left(E_{\ddagger}\right)\right]^{*}} \\
& =K\left\|\tilde{\mu}_{E}\right\|_{\left[\mathcal{W}_{\infty}\left(E_{\ddagger}\right)\right]^{*}} \leq K\left\|\mu_{E}\right\|_{\mathcal{M}} \text {. }
\end{aligned}
$$

We then have

$$
\left\|f_{E}-\mu_{E}\right\|_{\mathcal{M}^{\lambda}}=\left\|f_{E}-\mu_{E}\right\|_{\mathcal{M}^{\lambda}(E)}=\sup \left\{\left\langle f_{E}-\mu_{E}, w\right\rangle: w \in B(E)\right\}
$$

where $B(E)=\{w \in C(E):|w| \leq 1,|w(x)-w(y)| \leq|x-y|$ for $x, y \in E\}$. Given any $w \in B(E)$, we pick $x_{E} \in E$ and set $w_{0} \equiv w\left(x_{E}\right)$ so, by definition,

$$
\left|w(x)-w_{0}(x)\right|=\left|w(x)-w\left(x_{E}\right)\right| \leq\left|x-x_{E}\right|^{\lambda} \leq \operatorname{diam}(E)^{\lambda} \leq K h^{\lambda}
$$

- i.e., $\left\|w-w_{0}\right\|_{C(E)} \leq K h^{\lambda}$. Since the constant function $w_{0}$ is in $\mathcal{W}(E)$, we have $\left\langle f_{E}-\mu_{E}, w_{0}\right\rangle=0$ by the definition of $f_{E}$ whence

$$
\begin{aligned}
\left\langle f_{E}-\mu_{E}, w\right\rangle & =\left\langle f_{E}-\mu_{E}, w-w_{0}\right\rangle \\
& \leq\left\|f_{E}-\mu_{E}\right\|_{\mathcal{M}}\left\|w-w_{0}\right\|_{C(E)} \leq K h^{\lambda}\left\|\mu_{E}\right\|_{\mathcal{M}}
\end{aligned}
$$


since $\left\|f_{E}-\mu_{E}\right\|_{\mathcal{M}} \leq\left\|f_{E}\right\|_{\mathcal{M}}+\left\|\mu_{E}\right\|_{\mathcal{M}} \leq K\left\|\mu_{E}\right\|_{\mathcal{M}}$. The triangle inequality then gives $(17-c)$, completing the proof.

For the elliptic problem (2) we then easily obtain the anticipated convergence order:

Theorem 4.2 For dimensions $d=2,3$ and any $0 \leq \lambda<\alpha_{*}=2-d / 2$ for which $(7)$, (8) hold, there is a constant $K$ such that one has the $L^{2}(\Omega)$ error bound

$$
\|u-U\|_{L^{2}(\Omega)} \leq K h^{\lambda}\|\mu\|_{\mathcal{M}(\Omega)} .
$$

Here, for an arbitrary measure in $\mathcal{M}(\Omega)=[C(\Omega)]^{*}$, we have the solution $u=\mathbf{S} \mu \in L^{2}(\Omega)$ of (2) and the corresponding FEM solution $U=\mathbf{S}^{\mathcal{V}} \mu$, obtained by (6) for any regular triangulation $\mathcal{T} \in \mathfrak{T}_{h}$.

[We emphasize that $K$ in (27) depends on $\Omega$ and on the choice of $\lambda$ (presumably with $K \nearrow \infty$ as $\lambda \nearrow \alpha_{*}$ ), but not on the particular $\mu$ or on the particular triangulation used, subject to $A_{E} \in \mathfrak{A}$.]

Proof: $\quad$ By Theorem 4.1 we can introduce $f=\Pi^{\mathcal{V}} \mu \in L^{2}(\Omega)$, satisfying (17). By (17-a) one then has the same right hand side in (6) for $\varphi=f$ as for $\varphi=\mu$ so these give the same finite element solution: $U=\mathbf{S}^{\mathcal{V}} \mu=\mathbf{S}^{\mathcal{V}} f$ — although $\tilde{u}=\mathbf{S} f$ will not be the same as $u=\mathbf{S} \mu$. We now use (8) for $\mathbf{S}: \mathcal{X}^{-}=\mathcal{M}^{\lambda}(\Omega) \rightarrow L^{2}(\Omega)$ so, using (17-c), we do have

$$
\|u-\tilde{u}\|=\|\mathbf{S}[\mu-f]\| \leq K\|f-\mu\|_{\mathcal{X}^{-}} \leq K h^{\lambda}\|\mu\|_{\mathcal{M}(\Omega)} .
$$

Using (4) with (17-b) now gives

$$
\|\tilde{u}-U\|=\left\|\mathbf{S} f-\mathbf{S}^{\mathcal{V}} f\right\| \leq K h^{2}\|f\| \leq K h^{2-d / 2}\|\mu\|_{\mathcal{M}} .
$$

Finally, combining (28) with (29),

$$
\|u-U\| \leq\|u-\tilde{u}\|+\|\tilde{u}-U\| \leq K h^{\lambda}\|\mu\|_{\mathcal{M}}+K h^{2-d / 2}\|\mu\|_{\mathcal{M}}
$$

which just gives (27) for $\lambda<\alpha_{*}$.

\section{The parabolic problem}

Our goal in this section is to show that the same approximation theorems just used for FEM solution of the elliptic problem (2) also provide convergence orders for FEM semidiscretization of the parabolic problem (1). [For results on full discretization - in time as well as space - we refer to [12] with such minor adaptation as may be made necessary for the setting here.]

For the parabolic problem (1) the results obtainable depend, to a large extent, on what is assumed about the right-hand side as a function: $[0, T] \rightarrow \mathcal{M}$. 
For simplicity, we consider the problem here in continuous time (semidiscretization, corresponding to application of the Trotter-Kato Theorem) so the finite element solution $U=U(t)$ for (1) is given, much as in (6), by

$$
U(t) \in \mathcal{V}, \quad U(0)=0, \quad\left\langle U^{\prime}, v\right\rangle+\langle\nabla U, \nabla v\rangle=\langle\varphi, v\rangle \quad \text { for all } v \in \mathcal{V}
$$

for each $t \in[0, T]$. As with Theorem 4.2, we will use a known finite element error estimate $\left\|\mathbf{S}-\mathbf{S}_{\mathcal{V}}\right\| \leq K h^{2}$ (cf.,e.g., [12]) for $L^{2}$-valued data $\varphi$; more precisely, given some $p \in[0, \infty]$ and any $\varphi \in L^{p}\left([0, T] \rightarrow L^{2}(\Omega)\right)$, one has for $\mathcal{V} \in \mathfrak{V}_{h}$

$$
\|u-U\|_{L^{p}\left([0, T] \rightarrow L^{2}(\Omega)\right)} \leq K h^{2}\|\varphi\|_{L^{p}\left([0, T] \rightarrow L^{2}(\Omega)\right)} .
$$

We will also need some standard information regarding the equation (1). For this we use a semigroup formulation, noting that the unbounded operator $\mathbf{A}=(-\Delta)$ on $L^{2}(\Omega)$ — with domain $\mathcal{D}=H^{2}(\Omega) \cap H_{0}^{1}(\Omega)$ - is selfadjoint and positive definite so $\mathbf{- A}$ is the infinitesimal generator of an analytic semigroup $\mathbf{S}(\cdot)$ on $L^{2}(\Omega)$. Thus, the mild solution of $(1)$ is given by

$$
u(t)=\int_{0}^{t} \mathbf{S}(t-s) \varphi(s) d s
$$

provided $\varphi(s) \in L^{2}(\Omega)$ and the integral in (32) is well defined. For fractional powers of $\mathbf{A}$ (i.e., $\mathbf{A}^{\sigma}$ with $\sigma \geq 0$ ), we note (7) and the estimate for analytic semigroups

$$
\left\|\mathbf{A}^{\sigma} \mathbf{S}(t)\right\| \leq K t^{-\sigma} \quad \text { for } 0<t \leq T
$$

and also note in the present case (cf., [3], [7]) that the domain $\mathcal{D}\left(\mathbf{A}^{\sigma}\right)$ (say, for $1 / 2<\sigma<1$ so the boundary condition is relevant) is $H^{2 \sigma}(\Omega) \cap H_{0}^{1}(\Omega)$. We then have

Lemma 5.1 Given (7) there is $\sigma<1$ such that $H^{2 \sigma}(\Omega) \hookrightarrow C^{0, \lambda}(\Omega)$ so we have $\mathbf{A}^{-\sigma}: L^{2}(\Omega) \rightarrow C^{0, \lambda}(\Omega)$. By duality and selfadjointness we then also have

$$
\left\|\mathbf{A}^{-\sigma} \mu\right\|_{L^{2}(\Omega)} \leq K\|\mu\|_{\mathcal{M}^{\lambda}(\Omega)} .
$$

We now turn to $(1)$ with $\varphi=\mu(\cdot) \in L^{p}\left([0, T] \rightarrow \mathcal{M}^{\lambda}(\Omega)\right)$. Choosing $\sigma<1$ as in Lemma 5.1, we can rewrite (32) as

$$
u(t)=\int_{0}^{t}\left[\mathbf{A}^{\sigma} \mathbf{S}(t-s)\right]\left[\mathbf{A}^{-\sigma} \mu(s)\right] d s
$$

and (33) gives

$$
\|u(t)\|_{L^{2}(\Omega)} \leq K \int_{0}^{t}(t-s)^{-\sigma}\|\mu(s)\|_{\mathcal{M}^{\lambda}(\Omega)} d s .
$$

We recognize $(35)$ as bounding $\|u(\cdot)\|$ by the convolution of the integrable function $t^{-\sigma}$ and the $L^{p}$ function $\|\mu(\cdot)\|_{\mathcal{M}^{\lambda}}$, hence bounding $\|u(\cdot)\|$ in $L^{\hat{p}}(0, T)$ (for $p<\hat{p}<p /[1-(1-\sigma) p]$ ) by a standard convolution estimate to get

$$
\|u\|_{L^{\hat{p}}\left([0, T] \rightarrow L^{2}(\Omega)\right)} \leq K\|\mu\|_{L^{p}\left([0, T] \rightarrow \mathcal{M}^{\lambda}(\Omega)\right)} .
$$


Theorem 5.1 For dimensions $d=2,3$ and any $0 \leq \lambda<\alpha_{*}=2-d / 2$ for which we have $(7)$, one has the $L^{p}\left([0, T] \rightarrow L^{2}(\Omega)\right)$ error bound (for each $1 \leq p \leq \infty)$

$$
\|u-U\|_{L^{p}\left([0, T] \rightarrow L^{2}(\Omega)\right)} \leq K h^{\lambda}\|\mu\|_{L^{p}([0, T] \rightarrow \mathcal{M}(\Omega))}
$$

for an arbitrary measure-valued function $\mu(\cdot) \in L^{p}([0, T] \rightarrow \mathcal{M}(\Omega))$. Here u is the solution $\mathbf{S} * \mu$ of (1) with $\varphi=\mu$ and $U=\mathbf{S}^{\mathcal{V}} * \mu$ is the corresponding FEM solution, obtained by (30) for regular triangulations $\mathcal{T} \in \mathfrak{T}_{h}$.

[Again we emphasize that $K$ in (37) will depend on the choice of $\lambda<\alpha_{*}$.]

ProOF: As in the proof of Theorem 4.2 we introduce $f$, obtained pointwise in $t$ from $\mu$. We did not assert any continuity or linearity of $\Pi^{\mathcal{V}}$ in Theorem 4.1, but it is easy to see from the construction that the function $[t \mapsto f(t)]$ can be taken measurable whence $(17-b)$, gives $f \in L^{p}\left([0, T] \rightarrow L^{2}(\Omega)\right.$ and, by $(17-a)$, the construction in the Finite Element Method gives the same right hand side in (30) for $\varphi=f$ as for $\varphi=\mu$ so these give the same finite element solution $U$, although the solution $\tilde{u}$ of (1) is not $u$. Applying (36) to the difference $\tilde{u}-u$, and using the known error estimate $(31)$ along with $(17-b, c)$ then gives

$$
\begin{aligned}
\|u-U\|_{L^{p}\left([0, T] \rightarrow L^{2}(\Omega)\right)} & \\
& \leq\|\tilde{u}-u\|_{L^{p}\left([0, T] \rightarrow L^{2}(\Omega)\right)}+\|\tilde{u}-U\|_{L^{p}\left([0, T] \rightarrow L^{2}(\Omega)\right)} \\
& \leq K\|f-\mu\|_{L^{p}\left([0, T] \rightarrow \mathcal{M}^{\lambda}(\Omega)\right)}+K h^{2}\|f\|_{L^{p}\left([0, T] \rightarrow L^{2}(\Omega)\right)} \\
& \leq K\left(h^{\lambda}+h^{\alpha_{*}}\right)\|\mu\|_{L^{p}([0, T] \rightarrow \mathcal{M}(\Omega))}
\end{aligned}
$$

and so (37) as desired.

[Note also that taking $\mu \in \mathcal{M}(\mathcal{Q})=\mathcal{M}([0, T] \rightarrow \mathcal{M}(\Omega))$ would correspond, roughly, to taking $p=1$ in Theorem 5.1.]

Finally, we note that the sup-norm estimate for $p=\infty$ here gives a convergence order estimate pointwise in $t$ : For dimensions $d=2,3$ and any $0 \leq \lambda<\alpha_{*}=2-d / 2$, one has

$$
\begin{aligned}
\|u(t)-U(t)\|_{L^{2}(\Omega)} & \leq C h^{\lambda} \quad \text { for each } t \in[0, T] \\
\text { with } \quad C & =K\|\mu\|_{L^{\infty}([0, T] \rightarrow \mathcal{M}(\Omega))} .
\end{aligned}
$$

[Again, $C, K$ depend on $\lambda$. By a density argument, we note that $u$ will be in $C\left([0, T] \rightarrow L^{2}(\Omega)\right)$ even for $\mu(\cdot) \in L^{\infty}([0, T] \rightarrow \mathcal{M}(\Omega))$, discontinuous in $t$.

Remark 5.1 It might seem plausible that if we were to have a measure-valued source term with support bounded away from $\partial \Omega$ or, somewhat more generally, taking values in $\mathcal{M}_{c}(\Omega)$, then, as in the elliptic case of Theorem 3.2, this convergence order could be improved somewhat from $\lambda<\alpha_{*}$ in (37), (39) to $\lambda=\alpha_{*}$ by using (10) instead of (17). Certainly, in the context of Remark 3.1 and [9], we can take $\sigma=1$ to replace Lemma 5.1 and the estimate (34) by

$$
\left\|\mathbf{A}^{-1} \mu\right\|_{L^{2}(\Omega)} \leq K\|\mu\|_{\Xi^{-2}(\Omega)}
$$


- however, we note that the convolution argument used above requires the integrability of $t^{-\sigma}$, so taking $\sigma<1$, thus leaving us with the estimate of Theorem 5.1 taking $\lambda<\alpha_{*}$.

\section{Computational results}

In this section, we provide results of numerical tests which illustrate the convergence result Theorem 5.1 of the previous Section 5 for the time-dependent linear parabolic problem (1). The analogous numerical tests of Theorem 4.2 of Section 4 for the stationary problem (2) are already included in [6].

Specifically, we consider the linear parabolic heat equation (1) with $D \equiv 1$ in $\Omega$, imposing homogeneous Dirichlet boundary conditions, $u=0$ on $\partial \Omega$, in the spatial dimensions $d=2,3$ of interest. The domain is chosen to be $\Omega=(-1,1)^{d} \subset \mathbb{R}^{d}$ with the initial condition $u=0$ for compatability with the boundary conditions in order to focus the numerical studies on the nonsmoothness of the source term. As a test problem, we used a single Dirac delta distribution as source term, constant in $t$ so $\varphi(\mathbf{x}, t)=\delta(\mathbf{x})$ for all $t \in[0, T]$, positioning the injection site at the center of the domain. On physical grounds, it is clear that the solution, starting with $u=0$, will develop a sharp spike at the injection site, growing over time as one unit of material per unit of time is injected by the model. The form (39) of Theorem 5.1 applies.

The domain $\Omega$ has piecewise linear boundary and so can be discretized exactly, using triangular elements in two dimensions and tetrahedra in three dimensions. The convergence studies employ a sequence of meshes with $h$ halved in each refinement, starting from a coarse initial mesh to allow as many refinements as possible. The shape of $\Omega$ enables us to have at each stage a mesh point where $\delta(\mathbf{x})$ is centered.

The initial computations [8], [5] used special-purpose code in C to implement the linear Lagrange finite elements. The more general tests of the convergence behavior of finite elements used the software package COMSOL Multiphysics (www.comsol.com), chosen for its convenience and reliability. See [13] for a tutorial introduction, then [6] for a numerical investigation of Lagrange elements also of higher than linear order and of different domain shapes for the elliptic problem (2); in effect these computational studies complement the analytical results in [11] and Theorem 4.2. Based on this, we also use COMSOL in this paper to extend those studies to the time-dependent problem (1); see [14] for more information on the techniques used.

Figures 1 (a) and (b) show log-log plots of the error $\|u(t)-U(t)\|_{L^{2}(\Omega)}$ vs. the reciprocal $1 / h$ for $d=2$ and 3 , respectively. The predicted slopes of $-\alpha_{*}$ ( -1.0 for $d=2$ and -0.5 for $d=3$, respectively) are shown as dashed lines in each plot. The three solid lines show observed convergence orders at three points in time $t=2,3,4$, whose observed slopes $\lambda$ clearly confirm the theoretical prediction that we may take $\lambda \approx \alpha_{*}$ in estimating the error.

In the form (39), we computed $C, \lambda$ by linear regression in the log-log representation for each time $t$ in these plots. This gives $\lambda \approx 0.97$ for the lines 


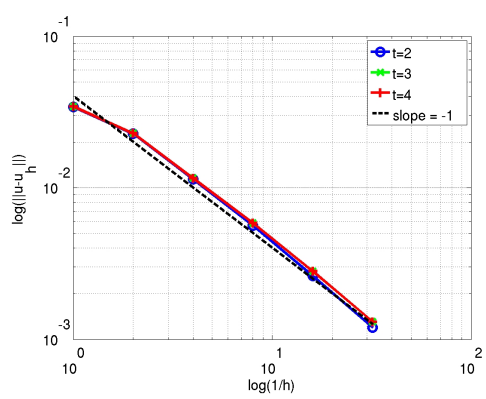

(a) $d=2$ triangular mesh

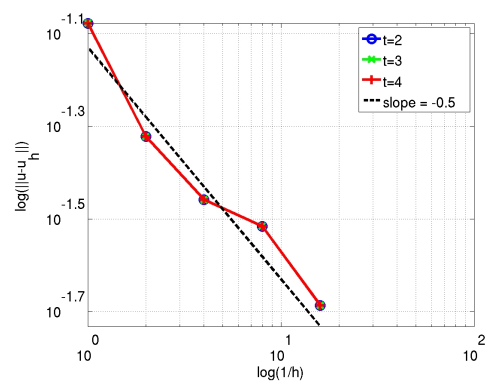

(b) $d=3$ tetragonal mesh

Fig. 1 Non-smooth test problem: $\log \left(\|u(t)-U(t)\|_{L^{2}(\Omega)}\right)$ vs. $\log (1 / h)$ at times $t=2,3,4$ and dashed line for predicted slope.

in $d=2$ dimensions and $\lambda \approx 0.47$ in $d=3$ dimensions. Both the plots and these quantitative results confirm the predicted analytic result, that the asymptotic convergence order is (approximately) given by $\alpha_{*}=2-d / 2$.

Acknowledgements The authors are indebted to Andreas Prohl for valuable input to the analysis of this paper.

The hardware used in the computational studies is part of the UMBC High Performance Computing Facility (HPCF). The facility is supported by the U.S. National Science Foundation through the MRI program (grant no. CNS-0821258) and the SCREMS program (grant no. DMS-0821311), with additional substantial support from the University of Maryland, Baltimore County (UMBC). See www.umbc.edu/hpcf for more information on HPCF and the projects using its resources. M. Kružík was partially supported by the grants IAA 100750802 (GA AV ČR), P201/10/0357, and P105/11/0411 (GA CR). D. W. Trott also acknowledges financial support as HPCF RA.

\section{References}

1. Casado-Díaz, J., Rebollo, T.C., Girault, V., Mármol, M.G., Murat, F.: Finite elements approximation of second order linear elliptic equations in divergence form with righthand side in $L^{1}$. Numer. Math. 105, 337-374 (2007)

2. Chrysafinos, K., Hou, L.S.: Error estimates for semidiscrete finite element approximations of linear and semilinear parabolic equations under minimal regularity assumptions. SIAM J. Numer. Anal. 40(1), 282-306 (2002)

3. Fujiwara, D.: Concrete characterization of the domains of fractional powers of some elliptic differential operators of the second order. Proc. Japan Acad. 43, 82-86 (1967)

4. Galouët, T.: Measure data and numerical schemes for elliptic problems. In: Elliptic and parabolic problems, Progr. Nonlinear Differential Equations Appl., vol. 63, pp. 279-290. Birkhäuser, Basel (2005)

5. Gobbert, M.K.: Long-time simulations on high resolution meshes to model calcium waves in a heart cell. SIAM J. Sci. Comput. 30(6), 2922-2947 (2008)

6. Gobbert, M.K., Yang, S.: Numerical demonstration of finite element convergence for Lagrange elements in COMSOL Multiphysics. In: V. Dravid (ed.) Proceedings of the COMSOL Conference 2008, Boston, MA (2008)

7. Grisvard, P.: Caractérisation de quelques espaces d'interpolation. Arch. Rational Mech. Anal. 26, 40-63 (1967)

8. Hanhart, A.L., Gobbert, M.K., Izu, L.T.: A memory-efficient finite element method for systems of reaction-diffusion equations with non-smooth forcing. J. Comput. Appl. Math. 169(2), 431-458 (2004) 
9. Lions, J.L., Magenes, E.: Non-homogeneous boundary value problems and applications. Vol. II. Springer-Verlag, New York (1972)

10. Rüde, U.: On the accurate computation of singular solutions of Laplace's and Poisson's equation. In: Multigrid methods (Copper Mountain, CO, 1987), Lecture Notes in Pure and Appl. Math., vol. 110, pp. 517-540. Dekker, New York (1988)

11. Scott, R.: Finite element convergence for singular data. Numer. Math. 21, 317-327 (1973)

12. Thomée, V.: Galerkin Finite Element Methods for Parabolic Problems, Springer Series in Computational Mathematics, vol. 25, second edn. Springer-Verlag (2006)

13. Trott, D.W., Gobbert, M.K.: Finite element convergence studies using COMSOL 4.0a and LiveLink for MATLAB. Tech. Rep. HPCF-2010-8, UMBC High Performance Computing Facility, University of Maryland, Baltimore County (2010). URL www.umbc.edu/hpcf

14. Trott, D.W., Gobbert, M.K.: Finite element convergence for time-dependent PDEs with a point source in COMSOL 4.2. In: Y. Rao (ed.) Proceedings of the COMSOL Conference 2011, Boston, MA (2011) 